\title{
A Novel Finite Element Technique for Moisture Diffusion Modeling using ANSYS
}

\author{
C. Diyaroglu, E. Madenci \\ Department of Aerospace and Mechanical Engineering \\ University of Arizona, Tucson, Arizona, USA \\ S. Oterkus, E. Oterkus \\ Department of Naval Architecture, Ocean and Marine Engineering \\ University of Strathclyde, Glasgow, UK
}

\begin{abstract}
This study presents a novel modeling approach for wetness and moisture concentration in the presence of time dependent saturated moisture concentration by employing the traditional ANSYS thermal and surface effect elements. The accuracy of the present approach is established by comparison with those of the existing ANSYS "diffusion" and "coupled field" elements as well as peridynamic theory. The comparison concerns the desorption process in a fully saturated bar made of two different materials with equal and unequal values of solubility activation energy in the presence of time dependent saturated moisture concentration under uniform and nonuniform temperature conditions. The results from the present approach agree well with those of peridynamics and ANSYS "coupled field" elements if the diffusivity is specified as time dependent. Significant deviation occurs if the diffusivity is specified as temperature dependent. The ANSYS "diffusion" element is applicable only for uniform temperature, and deviation becomes significant especially for unequal values of solubility activation energy.
\end{abstract}

\section{Introduction}

A large portion of microelectronic and optoelectronic components are made of polymeric materials including die attach materials, molding compound, underfill materials, etc. Polymeric materials are susceptible to moisture absorption which may yield significant amount of volume expansion. For multi-material systems as in electronic packages, such deformations can cause hygroscopic swelling and stresses. During the solder reflow process, moisture turns into highpressure vapor that can result in cracking coined as "popcorn cracking" [1]. Hence, it is essential to analyze the effect of moisture to ensure the reliability of the electronic packages.

As part of a finite element analysis, "wetness" concept introduced by Wong et al. [2] is commonly accepted for moisture concentration prediction in electronic packages with multi-material interfaces. Wetness approach is rather straightforward in the case of saturated concentration when independent of time and temperature. In such cases, a standard thermal-moisture analogy can be performed through a thermal diffusion analysis using a commercial software after calibrating its parameters. If saturated concentration depends on time, the wetness equation is no longer analogous to the thermal diffusion equation.

Wong et al. [3] extended the applicability of wetness concept for multi-material systems under transient loading and named it as "piecewise normalization" approach. However, this approach requires several load steps to ensure accuracy, and it is also computationally expensive due to its complex subroutines. For this reason, Wong [4] introduced another approach, which is more convenient and is easy to implement, named as "internal source" approach. However, the convergence of this approach is highly dependent on number of iterations carried out during each time step. Also, Diyaroglu et al. [5] applied peridynamics within the ANSYS framework to solve for wetness and moisture concentration in the presence of time dependent saturated moisture concentration without requiring iterative solution schemes.

As an alternative to these approaches, ANSYS, a commercial finite element software, offers "diffusion" and "coupled field" elements for wetness field and moisture concentration. These elements allow for either time or temperature dependent saturated moisture concentration. The ANSYS "diffusion" element is applicable only for uniform temperature. Although it permits time dependent saturated moisture concentration, the contribution from its rate of change is disregarded in the governing field equations.

This study presents a novel modeling approach for wetness and moisture concentration in the presence of time dependent saturated moisture concentration by employing the traditional ANSYS thermal and surface effect elements. Its accuracy is established by modeling the desorption process in a fully saturated bar made of two different materials with equal and unequal values of solubility activation energy in the presence of time dependent saturated moisture concentration under uniform and nonuniform temperature conditions. The present results agree well with those of ANSYS "coupled field" elements having time dependent diffusivity and peridynamics. Significant deviation occurs if the diffusivity is specified as temperature dependent. The ANSYS "diffusion" element leads to significant deviation especially for unequal values of solubility activation energy. This new approach removes the need for coupled field analysis and it is computationally efficient and easy to implement.

\section{Classical form of wetness equation}

Moisture diffusion can be described by the first Fick's law as

$\mathbf{J}=-D(t) \nabla C(t)$ 
where $C(t)$ is the moisture concentration, $\mathbf{J}$ is the diffusion flux vector and $D$ is the material diffusivity. During the diffusion process, conservation of the mass solute is satisfied by

$$
\nabla \cdot \mathbf{J}+\frac{\partial C}{\partial t}=G(t)
$$

in which $G(t)$ is the diffusing substance generation rate per unit volume. Substituting from Eq. (1) into Eq. (2) leads to

$$
\frac{\partial C}{\partial t}=D(t) \nabla \cdot(\nabla C)+G(t)
$$

This equation also known as the second Fick's law is valid only in homogeneous domains. In order to consider nonhomogeneous domains, Wong et al. [2] introduced a normalized wetness parameter, $\bar{C}$ in the form of

$$
\bar{C}=\frac{C(t)}{C_{s a t}(t)}
$$

in which $C_{\text {sat }}(t)$ is the saturated moisture concentration which defines the degree of saturation in a material. This normalization satisfies the equality of chemical potentials at the interface of dissimilar materials. According to this principle, the ratio of concentrations at the interface of materials $A$ and $B$ remains constant at any time as

$$
\frac{C_{A}}{C_{B}}=\frac{C_{\text {sat }, A} \bar{C}_{A}}{C_{\text {sat }, B} \bar{C}_{B}}=\text { constant }
$$

which results in $\bar{C}_{A}=\bar{C}_{B}$. Thus, the continuity of wetness automatically satisfies the equalization of chemical potentials at the interfaces.

Invoking the wetness parameter into Eq. (3) leads to the wetness equation as

$$
C_{s a t}(t) \frac{\partial \bar{C}}{\partial t}+\bar{C} \frac{\partial C_{s a t}(t)}{\partial t}=D \nabla \cdot\left(\nabla\left(C_{s a t} \bar{C}\right)\right)+G(t)
$$

For a constant, $C_{\text {sat }}$, this equation becomes

$$
C_{s a t} \frac{\partial \bar{C}}{\partial t}=D C_{s a t} \nabla \cdot(\nabla(\bar{C}))+G(t)
$$

Within the ANSYS framework, the solution to this equation can be directly constructed through the thermal analogy. However, the thermal analogy is not applicable when $C_{\text {sat }}$ is time dependent. Therefore, ANSYS element library offers "diffusion" elements and "coupled field" elements to solve for the wetness field in the presence of time dependent $C_{\text {sat }}[6]$.
However, these elements are based on the modified form of the first Fick's law as

$$
\mathbf{J}=-D(t) \nabla C(t)+\mathbf{v} C(t)
$$

and

$$
\mathbf{J}=-D(t) \nabla C(t)-\frac{D(t) Q}{k} \frac{1}{T^{2}} C(t) \nabla T(t)
$$

in which $\mathbf{v}$ represents the transport velocity vector, $Q$ is particle heat of transport, $k$ is Boltzmann constant. Substituting from Eq. (8a) into Eq. (2) leads to

$$
\begin{aligned}
C_{\text {sat }}(t) \frac{\partial \bar{C}}{\partial t}+\bar{C} \frac{\partial C_{s a t}(t)}{\partial t}= & D \nabla \cdot\left(\nabla\left(C_{s a t} \bar{C}\right)\right) \\
& -\nabla \cdot\left(\mathbf{v}\left(C_{s a t} \bar{C}\right)\right)+G(t)
\end{aligned}
$$

in which the unknown field is $\bar{C}(\mathbf{x}, t)$. Assuming the rate of saturated moisture concentration to be negligible, this equation can be reduced to a simpler form as

$C_{\text {sat }} \frac{\partial \bar{C}}{\partial t}=D C_{\text {sat }} \nabla \cdot \nabla \bar{C}-C_{\text {sat }} \nabla \cdot(\mathbf{v} \bar{C})+G(t)$

Similarly, substituting from Eq. (8b) into Eq. (2) leads to

$$
\begin{aligned}
& C_{s a t}(t) \frac{\partial \bar{C}}{\partial t}+\bar{C} \frac{\partial C_{s a t}}{\partial t}= \\
& D \nabla C_{s a t} \cdot(\nabla \bar{C})+D C_{s a t} \nabla \cdot(\nabla \bar{C}) \\
& +D \nabla \bar{C} \cdot\left(\nabla C_{\text {sat }}\right)+D \bar{C} \nabla \cdot\left(\nabla C_{\text {sat }}\right) \\
& +\frac{Q D C_{s a t} \bar{C}}{k} \nabla\left(\frac{1}{T^{2}}\right) \cdot(\nabla T)+\frac{Q D C_{s a t}}{k T^{2}} \nabla \bar{C} \cdot(\nabla T) \\
& +\frac{Q D \bar{C}}{k T^{2}} \nabla C_{s a t} \cdot(\nabla T)+\frac{Q D C_{s a t} \bar{C}}{k T^{2}} \nabla \cdot(\nabla T)+G
\end{aligned}
$$

If the saturated concentration is expressed as $C_{\text {sat }}(T)$ with $T(x, y, z, t)$ representing temperature, applying the chain rule permits this equation to be rewritten as

$$
\begin{aligned}
& C_{\text {sat }}(T) \frac{\partial \bar{C}}{\partial t}+\bar{C} \frac{\partial C_{s a t}(T)}{\partial T} \frac{\partial T}{\partial t}= \\
& D \frac{\partial C_{s a t}}{\partial T} \nabla T \cdot \nabla \bar{C}+D C_{s a t}(T) \nabla \cdot \nabla \bar{C} \\
& +D \frac{\partial C_{s a t}}{\partial T} \nabla \bar{C} \cdot \nabla T+D \bar{C} \frac{\partial C_{s a t}}{\partial T} \nabla \cdot \nabla T \\
& -\frac{2 D Q C_{s a t} \bar{C}}{k} \frac{1}{T^{3}} \nabla T \cdot(\nabla T)+\frac{D Q C_{s a t}}{k T^{2}} \nabla \bar{C} \cdot(\nabla T) \\
& +\frac{D Q \bar{C}}{k T^{2}} \frac{\partial C_{s a t}}{\partial T} \nabla T \cdot(\nabla T)+\frac{D Q C_{s a t} \bar{C}}{k T^{2}} \nabla \cdot(\nabla T)+G
\end{aligned}
$$


in which the unknown fields are $\bar{C}(\mathbf{x}, t)$ and $T(\mathbf{x}, t)$. Therefore, it is referred to as coupled wetness field equation. In this, equation, diffusivity can be specified as time or temperature dependent, i.e., $D=D(t)$ or $D=D(T)$.

\section{ANSYS "diffusion' and "coupled field" elements}

ANSYS, a commercial finite element software, offers "diffusion" elements (Plane 238 with 8 nodes, Solid 239 with 20 nodes and Solid 240 with 10 nodes) with only one degree of freedom (DOF) as concentration (CONC) at each node. These elements are suitable for the solution of Eq. (10) based on constant $C_{\text {sat }}$ approximation. If $C_{\text {sat }}$ is defined with "MP, CSAT" command in ANSYS, the concentration DOF represents the wetness parameter. This element permits either time or temperature dependent $C_{\text {sat }}$ values . However, it is suitable for only uniform temperature. In the case of nonuniform temperature, ANSYS offers "coupled field" elements (Plane 223 with 8 nodes, Solid 226 with 20 nodes and Solid 227 with 10 nodes) with two DOF as wetness, $\bar{C}$ and temperature, $T$ at each node. The "coupled field" elements enable the modeling of temperature dependent conditions; thus, suitable for the solution of Eq. (12). Their approximation over element " $e$ " can be achieved by

$\bar{C}=\mathbf{N} \cdot \mathbf{C}_{e}$

and

$T=\mathbf{N} \cdot \mathbf{T}_{e}$

where $\mathbf{C}_{e}$ and $\mathbf{T}_{e}$ are the vectors of unknown wetness and temperature at the nodes, and $\mathbf{N}$ is the vector of element shape functions. Their nodal rates are denoted by $\dot{\mathbf{C}}_{e}$ and $\dot{\mathbf{T}}_{e}$. Applying the virtual work principle over the element " $e$ " with arbitrary virtual quantities of $\mathbf{C}_{e}$ and $\mathbf{T}_{e}$, Eq. (12) can be rewritten as

$$
\begin{aligned}
\mathbf{C}^{d t} \dot{\mathbf{T}}_{e}+\mathbf{C}^{d} \dot{\mathbf{C}}_{e} & =\left(\mathbf{K}^{d}+\mathbf{K}_{1}^{d N}+\mathbf{K}_{2}^{d N}\right) \mathbf{C}_{e} \\
& +\left(\mathbf{K}^{d t N}+\mathbf{K}_{1}^{d t}+\mathbf{K}_{2}^{d t}+\mathbf{K}_{2}^{d t N}\right) \mathbf{T}_{e}+\mathbf{R}
\end{aligned}
$$

where $\mathbf{C}^{d}$ is the element diffusion damping matrix, $\mathbf{C}^{d t}$ is the element thermal-diffusion damping matrix, $\mathbf{K}^{d}$ is the element diffusion conductivity matrix, $\mathbf{K}_{1}^{d N}$ is the nonlinear part of the element diffusion conductivity matrix associated with thermomigration, $\mathbf{K}_{2}^{d N}$ is the nonlinear part of the element diffusion conductivity matrix produced by $\partial C_{\text {sat }} / \partial T$, $\mathbf{K}^{d t N}$ is the nonlinear part of the element transport conductivity matrix, $\mathbf{K}_{1}^{d t}$ is the element transport conductivity matrix, $\mathbf{K}_{2}^{d t}$ is the element thermal - diffusion conductivity matrix produced by $\partial C_{\text {sat }} / \partial T, \mathbf{K}_{2}^{d t N}$ is the nonlinear part of the element transport conductivity matrix produced by $\partial C_{\text {sat }} / \partial T$, and $\mathbf{R}$ is the nodal diffusion flow rate vector. They are explicitly defined in ANSYS manual [6] as

$\mathbf{C}^{d}=C_{s a t}(T) \int_{V_{e}} \mathbf{N N}^{\mathbf{T}} d V$

$\mathbf{C}^{d t}=\frac{\partial C_{\text {sat }}}{\partial T} \int_{V_{e}} \bar{C} \mathbf{N N}^{\mathbf{T}} d V$

$\mathbf{K}^{d}=-D C_{\text {sat }}(T) \int_{V_{e}}\left(\nabla \mathbf{N}^{T}\right)^{T} \nabla \mathbf{N}^{T} d V$

$\mathbf{K}^{d t N}=-2 D C_{s a t}(T) \frac{Q}{k} \int_{V_{e}} \frac{\bar{C}}{T^{3}}\left(\nabla \mathbf{N}^{T}\right)^{T}\left(\nabla T \mathbf{N}^{T}\right) d V$

$\mathbf{K}_{1}^{\mathrm{dt}}=-D C_{\text {sat }}(T) \frac{Q}{k} \int_{V_{e}} \frac{\bar{C}}{T^{2}}\left(\nabla \mathbf{N}^{T}\right)^{T} \nabla \mathbf{N}^{T} d V$

$\mathbf{K}_{1}^{d N}=D C_{s a t}(T) \frac{Q}{k} \int_{V_{e}} \frac{1}{T^{2}}\left(\nabla \mathbf{N}^{T}\right)^{T}\left(\nabla T \mathbf{N}^{T}\right) d V$

$\mathbf{K}_{2}^{d t}=-D \frac{\partial C_{\text {sat }}}{\partial T} \int_{V_{e}} \bar{C}\left(\nabla \mathbf{N}^{T}\right)^{T} \nabla \mathbf{N}^{T} d V$

$\mathbf{K}_{2}^{d N}=D \frac{\partial C_{\text {sat }}}{\partial T} \int_{V_{e}}\left[\left(\nabla \mathbf{N}^{T}\right)^{T}\left(\mathbf{N}(\nabla T)^{T}\right)+\left(\nabla \mathbf{N}^{T}\right)^{T}\left(\nabla T \mathbf{N}^{T}\right)\right] d V(15 \mathrm{~h})$

$\mathbf{K}_{2}^{d t N}=D \frac{\partial C_{\text {sat }}}{\partial T} \frac{Q}{k} \int_{V_{e}} \frac{\bar{C}}{T^{2}}\left(\nabla \mathbf{N}^{T}\right)^{T}\left(\nabla T \mathbf{N}^{T}\right) d V$

$\mathbf{R}=G \int_{V_{e}} \mathbf{N} d V$

As described in the ANSYS manual [6], the final form of the coupled finite element thermal-diffusion matrix equations can be expressed as

$$
\begin{aligned}
& {\left[\begin{array}{cc}
\mathbf{C}^{t} & \mathbf{0} \\
\mathbf{C}^{d t} & \mathbf{C}^{d}
\end{array}\right]\left\{\begin{array}{c}
\dot{\mathbf{T}}_{e} \\
\dot{\mathbf{C}}_{e}
\end{array}\right\}+} \\
& {\left[\begin{array}{cc}
\mathbf{K}^{t} & \mathbf{0} \\
\left(\mathbf{K}_{1}^{d t}+\mathbf{K}^{d t N}+\mathbf{K}_{2}^{d t N}+\mathbf{K}_{2}^{d t}\right) & \left(\mathbf{K}^{d}+\mathbf{K}_{1}^{d N}+\mathbf{K}_{2}^{d N}\right)
\end{array}\right]\left\{\begin{array}{c}
\mathbf{T}_{e} \\
\mathbf{C}_{e}
\end{array}\right\}} \\
& =\left\{\begin{array}{l}
\mathbf{Q} \\
\mathbf{R}
\end{array}\right\}
\end{aligned}
$$

in which $\mathbf{C}^{t}, \mathbf{K}^{t}$ and $\mathbf{Q}$ are relevant only to the heat flow representing the element specific heat matrix, the element thermal conductivity matrix and the element heat generation load matrix, respectively. The thermal conductivity matrix can be expressed as $\mathbf{K}^{t}=\mathbf{K}^{t m}+\mathbf{K}^{t b}+\mathbf{K}^{t c}$ in which $\mathbf{K}^{t m}, \mathbf{K}^{t b}$ 
and $\mathbf{K}^{\text {tc }}$ are the element mass transport conductivity matrix, element diffusion conductivity matrix and element convection surface conductivity matrix, respectively. If the thermomigration is negligible, this equation can be reduced to

$$
\left[\begin{array}{cc}
\mathbf{C}^{t} & \mathbf{0} \\
\mathbf{C}^{d t} & \mathbf{C}^{d}
\end{array}\right]\left\{\begin{array}{c}
\dot{\mathbf{T}}_{e} \\
\dot{\mathbf{C}}_{e}
\end{array}\right\}+\left[\begin{array}{cc}
\mathbf{K}^{t} & \mathbf{0} \\
\mathbf{K}_{2}^{d t N} & \left(\mathbf{K}^{d}+\mathbf{K}_{2}^{d N}\right)
\end{array}\right]\left\{\begin{array}{c}
\mathbf{T}_{e} \\
\mathbf{C}_{e}
\end{array}\right\}=\left\{\begin{array}{c}
\mathbf{Q} \\
\mathbf{R}
\end{array}\right\}
$$

\section{ANSYS thermal and surface effect elements}

The traditional ANSYS thermal element and surface effect element can be employed for solving the wetness equation, Eq. (6) with $D=D(t)$ and $C_{\text {sat }}=C_{\text {sat }}(t)$. Applying the virtual work principle and using the divergence theorem, Eq. (6) can be rewritten as

$$
\begin{aligned}
& \delta \bar{C} \int_{V_{e}} C_{s a t}(t) \frac{\partial \bar{C}}{\partial t} d V+\delta \bar{C} \int_{V_{e}} \bar{C} \frac{\partial C_{s a t}}{\partial t} d V= \\
& -D(t) C_{s a t}(t) \int_{V_{e}} \nabla(\delta \bar{C}) \nabla \bar{C} d V \\
& +D(t) C_{s a t}(t) \delta \bar{C} \int_{V_{e}} G(\mathbf{x}, t) d V+\delta \bar{C} \int_{S_{e}} \nabla \bar{C} \mathbf{n} d S
\end{aligned}
$$

where $V_{e}, S_{e}$ and $\mathbf{n}$ indicate the element's volume, surface and unit normal to the surface, respectively. In this equation, the rate of change of $C_{\text {sat }}(t)$, i.e. $d C_{\text {sat }} / d t$, is approximated by using backward Euler method as

$$
H=\bar{C} \frac{\Delta C_{\text {sat }}}{\Delta t}
$$

After substituting for $\bar{C}$ in Eq. (18) from Eq. (13a) with arbitrary $\delta \mathbf{C}_{e}$, Eq. (18) can be rewritten in a matrix form

$$
\left(\mathbf{C}^{d t}+\mathbf{C}^{d}\right) \dot{\mathbf{C}}_{e}+\mathbf{K}^{d} \mathbf{C}_{\mathbf{e}}=\mathbf{R}
$$

where $\mathbf{C}^{d t}, \mathbf{C}^{d}$ and $\mathbf{K}^{d}$ are expressed as

$$
\begin{aligned}
& \mathbf{C}^{d}=C_{\text {sat }}(t) \int_{V_{e}} \mathbf{N N}^{\mathbf{T}} d V \\
& \mathbf{K}^{d}=D C_{s a t}(t) \int_{V_{e}}\left(\nabla \mathbf{N}^{T}\right)^{T} \nabla \mathbf{N}^{T} d V \\
& \mathbf{C}^{d t}=\frac{\partial C_{s a t}}{\partial t} \int_{V_{e}} \mathbf{N N}^{\mathbf{T}} d V \\
& \mathbf{R}=G \int_{V_{e}} \mathbf{N} d V
\end{aligned}
$$

This equation is analogous to the heat flow equation described in ANSYS as
$\mathbf{C}_{e}^{t} \dot{\mathbf{T}}_{e}+\left(\mathbf{K}_{e}^{t b}+\mathbf{K}_{e}^{t c}\right) \mathbf{T}_{e}=\mathbf{Q}_{e}^{g}$

where $\mathbf{C}_{e}^{t}, \mathbf{K}_{e}^{t b}, \mathbf{K}_{e}^{t c}$ and $\mathbf{Q}_{e}^{g}$ are element specific heat matrix, element diffusion conductivity matrix, element convection surface conductivity matrix and element heat generation load, respectively. They are explicitly defined as

$$
\mathbf{C}_{e}^{t}=\rho c \int_{V} \mathbf{N N}^{\mathbf{T}} d V
$$

$\mathbf{K}_{e}^{t b}=D_{t} \int_{V}\left(\nabla \mathbf{N}^{T}\right)^{T} \nabla \mathbf{N}^{T} d V$

$\mathbf{K}_{e}^{t c}=h_{f} \int_{S} \mathbf{N N}^{T} d S$

$\mathbf{Q}_{e}^{g}=\dddot{q} \int_{V} \mathbf{N} d V$

where $\rho, c, D(t), h_{f}$ and $\dddot{q}$ indicate the density, the specific heat, the thermal conductivity, the film coefficient and the heat generation rate per unit volume, respectively. Comparing the wetness and heat flow equations with each other, i.e., Eqs. (20) and (22), there exists correspondence between the thermal parameters, $\rho c, D_{t}, h_{f}$ and $\dddot{q}$ and diffusion parameters, $C_{\text {sat }}(t), D C_{\text {sat }}(t), \partial C_{\text {sat }} / \partial t$ and $G$. Therefore, Eq. (20) can be easily constructed by modifying variables in ANSYS as demonstrated for one-dimensional analysis in Tables 1 and 2 .

For one-dimensional analysis, this equation can be constructed by using thermal link (LINK33) and thermal surface effect (SURF151) elements between two nodes, shown in Fig 1. The thermal link element is used for $\mathbf{C}_{e}^{t}$ and $\mathbf{K}_{e}^{t b}$ matrices and the thermal surface effect for $\mathbf{K}_{e}^{t c}$ matrix. The modification of thermal parameters with diffusion parameters for LINK33 and SURF151 elements are demonstrated in Tables 1 and 2. In Table 1, the subscript $n$ at $D_{n+1}$ and $C_{s a t, n+1}$ specifies the current time step number. In Table 2, the rate of $C_{\text {sat }}$ is approximated with backward Euler method as $\Delta C_{\text {sat }} / \Delta t$ in which $\Delta$ implies the difference of values between the time steps. Moreover, the nodal heat generation rate per unit volume, $\dddot{q}$, can be used in place of diffusing substance generation rate per unit volume, $G$, in ANSYS by defining the nodal loads with "BF, Node, HGEN, Value" command.

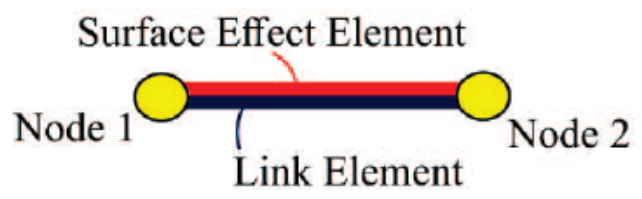

Fig. 1. Thermal link and surface effect elements between nodes 1 and 2 


\section{Numerical results}

The numerical results concern the modeling of desorption process in a bar by employing the traditional thermal link and surface effect elements, "diffusion" elements and "coupled field" elements. The bar is composed of two different materials as shown in Fig. 2. It is initially fully saturated at $85^{\circ} \mathrm{C} / 100 \% R H$ with isolated lateral surfaces. The properties of each material are given in Tables 3 and 4 previously considered by Wang et al. [7].

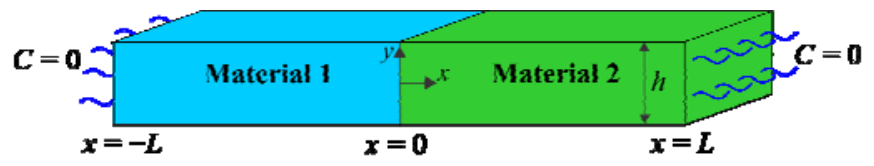

Fig. 2. A bar composed of two materials

The initial and boundary conditions in terms of the wetness parameter can be specified as

$$
\begin{aligned}
& \bar{C}(x, t=0)=1 \text { with }-L \leq x \leq L \\
& \bar{C}(x= \pm L, t)=0 \\
& \frac{\partial \bar{C}}{\partial y}(x, y= \pm h / 2)=0 \text { with }-h / 2 \leq y \leq h / 2
\end{aligned}
$$

in which $2 L$ and $h$ represent the length and thickness of the bar, respectively.

Also, the solubility activation energy of materials 1 and 2 can be either equal or unequal at the interface. The saturated concentration, $C_{s a t}(t)$ and diffusivity, $D(t)$ can be expressed as

$$
D(t)=D_{0} e^{\left(\frac{-E_{D}}{R T(t)}\right)}
$$

and

$$
C_{\text {sat }}(t)=S_{0} P_{0} e^{\left(\frac{E_{S}-E_{V P}}{R T(t)}\right)} \times R H
$$

in which $R$ is the universal gas constant ( $R=8.3145 \mathrm{~J} / \mathrm{Kmol}$ ), $D_{0}$ is the diffusivity factor, $S_{0}$ is the solubility factor, $P_{0}$ is the pressure factor, $E_{D}$ is the activation energy of the diffusivity, $E_{S}$ is the activation energy of the solubility, $E_{V P}$ is the vapor pressure activation energy, and $R H$ indicates the relative humidity.

In the case of "coupled field" elements, the saturated concentration, $C_{s a t}(T)$ is specifed as temperature dependent. However, the diffusivity can be specified as either time dependent, $D(t)$ or temperature dependent, $D(T)$. The temperature field can be either uniform or nonuniform. The uniform temperature is applied as
$T(x, t)=(85+2 t){ }^{\circ} \mathrm{C}$

The nonuniform temperature is achieved through the boundary conditions specified as

$T(x= \pm L, t)=(85+2 t){ }^{\circ} \mathrm{C}$

However, the "diffusion" element is not applicable under nonuniform temperature because the temperature is not known apriori. Also, the solutions with "diffusion" and "coupled field" elements are obtained for negligible transport velocity and thermomigration, respectively.

The FE discretization of a bar with thermal elements and with 2D "diffusion" or "coupled field" elements are shown in Figs. 3 and 4 . The bar has a length of $2 L=2 \mathrm{~mm}$ and a thickness of $h=L / 100$. Its cross sectional area is specified as $A=h^{2}$. The spacing between the nodes is specified as $\Delta x=h$. In modeling with of $1 \mathrm{D}$ thermal elements, the crosssectional area is defined by "R, NSET, $A$ " command and with 2D "diffusion" and "coupled field" elements only the thickness of the bar is defined by "R, NSET, $\Delta x$ " command. The solution time step size is chosen as $\Delta t=2 \mathrm{sec}$ and the total time is specified as $t=80 \mathrm{sec}$, and the moisture concentration results are plotted at $t=60 \mathrm{sec}$. The results obtained with the ANSYS thermal, diffusion and coupled field elements are also compared against the peridynamic solution [5] for uniform and nonuniform temperature with equal and unequal solubility activation energy of materials 1 and 2. In the case of unequal solubility activation energy, material 2 has $E_{S_{2}}=4.50 \times 10^{4} \mathrm{~J} / \mathrm{mol}$.

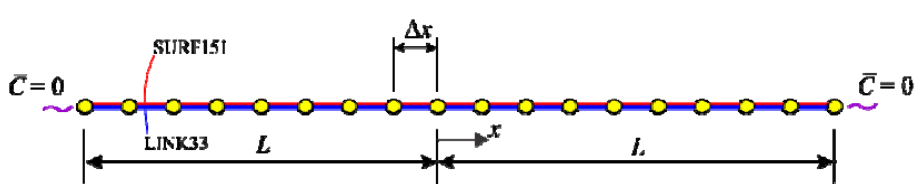

Fig. 3. The FE discretization of a bar with present approach

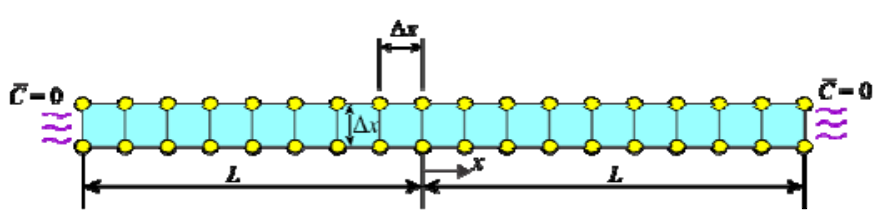

Fig. 4. The FE discretization of a bar with "diffusion" or "coupled field" elements

Desorption under uniform temperature with equal and unequal values of $E_{S}$

As shown in Figs. 5 and 6, the predictions based on the present approach with thermal elements, "coupled field" elements with $D(t)$ and $C_{\text {sat }}(T)$ and peridynamics are in 
excellent agreement for both equal and unequal values of $E_{S}$. The "coupled field" elements with $D(T)$ and $C_{\text {sat }}(T)$ and "diffusion" elements present significant deviation from the other solutions. When diffusivity, $D(t)$ is imposed as a time dependent property, its value is known before the next time step as part of time integration, and does not require approximation. However, it requires approximation for the next time step if imposed as $D(T)$ leading to the deviation in results. Therefore, the results deviate significantly from the correct solution. Also, the "diffusion" elements do not predict the correct concentration because it disregards the effect of rate of saturated concentration.

Desorption under nonuniform temperature with equal and unequal values of $E_{S}$

The "diffusion" element is not applicable due to the nonuniformity of temperature field. Also, the "coupled field" element with time dependent $D(t)$ is not applicable because diffusivity, $D(t)$ given in Eq. (25a) highly depends on temperature which is the unknown DOF in this problem. The concentration results along the bar are plotted in Figs. 7 and 8. As apparent from these figures, the predictions from the present approach and peridynamics are in excellent agreement. However, the results from the ANSYS "coupled field" elements with temperature dependent $D(T)$ deviate significantly.

\section{Conclusions}

This study presents a new modeling approach for wetness and moisture concentration in the presence of time dependent saturated moisture concentration by employing the traditional ANSYS thermal and surface effect elements. The accuracy of the present approach is established by comparison with those of the existing ANSYS "diffusion" and "coupled field" elements as well as peridynamic theory. The comparison concerns the desorption process in a fully saturated bar made of two different materials with equal and unequal values of solubility activation energy in the presence of time dependent saturated moisture concentration under uniform and nonuniform temperature conditions. The present approach predicts correct concentration for all cases considered. However, the accuracy of the predictions based on ANSYS "coupled field" elements depends on the applied temperature. Significant deviation occurs if the diffusivity is specified as temperature dependent. The ANSYS "diffusion" element is applicable only for uniform temperature, and deviation becomes significant especially for unequal values of solubility activation energy. This new computationally efficient and accurate approach removes the need for coupled field analysis.

\section{References}

1. Wong, E. H. and Park, B.,2016, "Moisture diffusion modeling - a critical review", Microelectronics Reliability, Vol. 65, pp. 318-326.
2. Wong, E. H., Teo, Y. C. and Lim, T. B.,1998, "Moisture diffusion and vapor pressure modeling of IC packaging," Proceedings of the 48th Electronic Components and Technology Conference, pp. 1372-1378.

3. Wong, E. H., Koh, S. W., Lee, K. H. and Rajoo, R.,2002, "Advanced moisture diffusion modeling \& characterization for electronic packaging," Proceedings of the 52nd Electronic Components and Technology Conference, pp. 1297-1303.

4. Wong, E.H., 2015, "The fundamentals of thermal-mass diffusion analogy,' Microelectronics Reliability, Vol 55, pp. $588-595$.

5. Diyaroglu, C., Oterkus, S., Oterkus, E., Madenci, E., Han, S. and Hwang, Y.,2017, "Peridynamic wetness approach for moisture concentration analysis in electronic packages. Microelectronics Reliability, Vol. 70, pp.103-111.

\section{ANSYS 19.0 Mechanical User's Guide, 2018.}

7. Wang, J., Liu, R., Liu, D., \& Park, S.,2017,“ Advancement in simulating moisture diffusion in electronic packages under dynamic thermal loading conditions," Microelectronics Reliability, Vol. 73, 42-53. 
Table 1. The correspondence of variables for thermal link element

\begin{tabular}{|c|c|c|c|c|}
\hline & & \multicolumn{3}{|r|}{ LINK33 } \\
\hline \multirow{4}{*}{$\begin{array}{c}\text { Material } \\
\text { Properties }\end{array}$} & & Original & Modified & ANSYS Command \\
\hline & Thermal Conductivity & $D_{t}$ & $D_{n+1} C_{s a t, n+1}$ & $\mathrm{MP}, \mathrm{KXX}, \mathrm{MAT}^{*}, D_{n+1} C_{s a t, n+1}$ \\
\hline & Density & $\rho$ & 1.0 & MP, DENS, MAT ${ }^{*}, 1.0$ \\
\hline & Specific Heat & $c$ & $C_{s a t, n+1}$ & MP, C, MAT ${ }^{*}, C_{s a t, n+1}$ \\
\hline $\begin{array}{c}\text { Real } \\
\text { Constants }\end{array}$ & Cross Sectional Area & $A$ & $A$ & $\mathrm{R}, \mathrm{NSET}^{*}, A$ \\
\hline
\end{tabular}

*MAT: Material reference number

*NSET: Real constant set identification number

Table 2. The correspondence for surface effect element

\begin{tabular}{|c|c|c|c|c|}
\hline & \multicolumn{3}{|c|}{ SURF151 } \\
\hline \multirow{2}{*}{$\begin{array}{c}\text { Applying Surface } \\
\text { loads on elements } \\
\text { (SFE) }\end{array}$} & Convection (CONV) & Original & Modified & ANSYS Command \\
\cline { 2 - 5 } & Film Coefficient & $h_{f}$ & $\frac{\Delta C_{\text {sat }} A}{\Delta t}$ & SFE, Elem ${ }^{*},$, CONV, $1, \frac{\Delta C_{s a t} A}{\Delta t}$ \\
\cline { 2 - 5 } & Bulk Temperature & $T_{B}$ & 0.0 & SFE, Elem*, CONV, $2,0.0$ \\
\hline
\end{tabular}

*Elem: Element to which surface load applies

Table 3. Diffusion Material Properties [7]

\begin{tabular}{|c|c|c|}
\hline & Material 1 & Material 2 \\
\hline Diffusivity factor, $D_{0}\left(\mathrm{~m}^{2} / \mathrm{s}\right)$ & $5 \times 10^{-3}$ & $4 \times 10^{-3}$ \\
\hline Solubility factor, $S_{0}\left(\mathrm{~kg} / \mathrm{m}^{3} \mathrm{~Pa}\right)$ & $6 \times 10^{-10}$ & $2 \times 10^{-10}$ \\
\hline Pressure factor, $P_{0}(\mathrm{~Pa})$ & $5.0492 \times 10^{10}$ & $5.0492 \times 10^{10}$ \\
\hline Diffusion activation energy, $E_{D}(\mathrm{~J} / \mathrm{mol})$ & $5 \times 10^{4}$ & $5 \times 10^{4}$ \\
\hline Solubility activation energy, $E_{S}(\mathrm{~J} / \mathrm{mol})$ & $4 \times 10^{4}$ & $4 \times 10^{4}$ \\
\hline Vapor pressure activation energy, $E_{V P}(\mathrm{~J} / \mathrm{mol})$ & $4.08737 \times 10^{4}$ & $4.08737 \times 10^{4}$ \\
\hline
\end{tabular}

Table 4. Thermal Material Properties [7]

\begin{tabular}{|c|c|c|}
\hline & Material 1 & Material 2 \\
\hline Density, $\rho\left(\mathrm{kg} / \mathrm{m}^{3}\right)$ & $3 \times 10^{3}$ & $3 \times 10^{3}$ \\
\hline Specific Heat, $c_{v}(\mathrm{~J} / \mathrm{kgK})$ & $1.5 \times 10^{3}$ & $1.5 \times 10^{3}$ \\
\hline Thermal Conductivity, $k(\mathrm{~W} / \mathrm{mK})$ & 0.6 & 0.6 \\
\hline
\end{tabular}




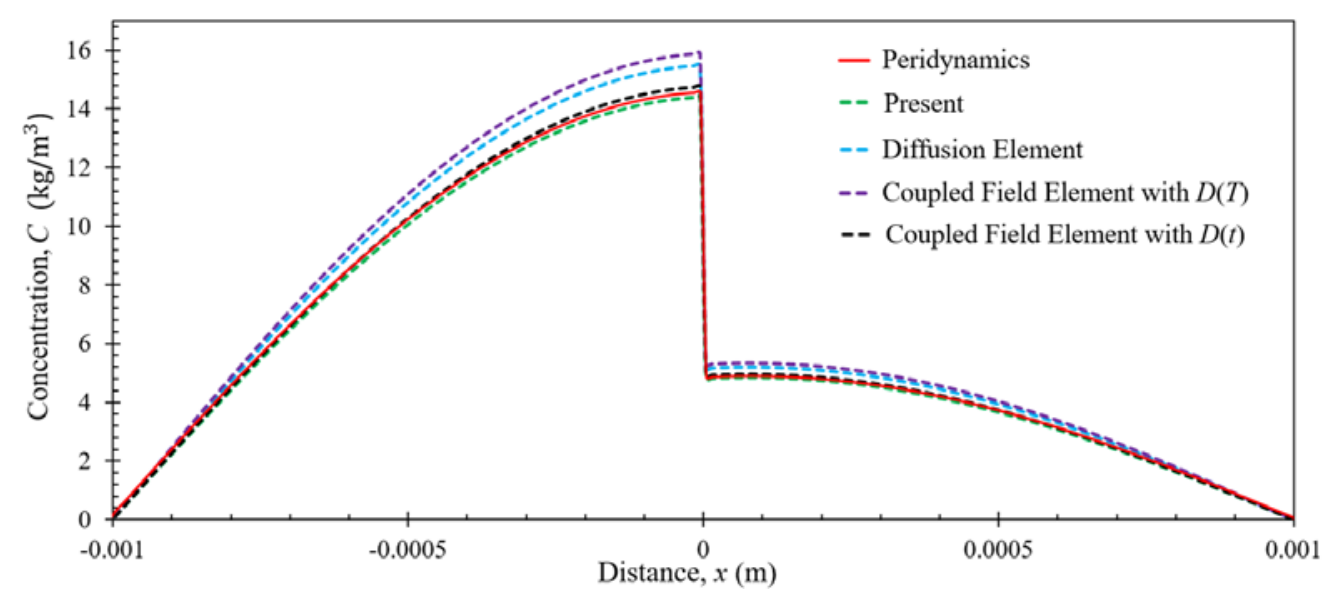

Fig. 5. Moisture concentration variation along the bar $t=60 \mathrm{sec}$ with equal $E_{S}$

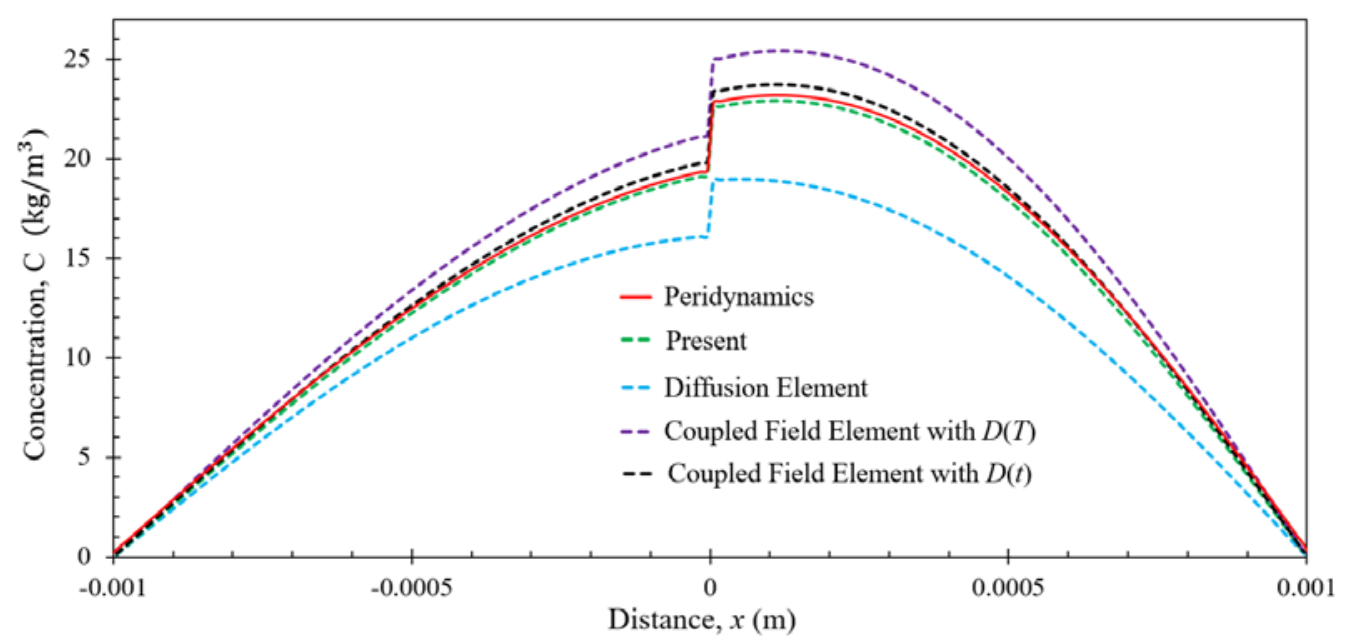

Fig. 6. Moisture concentration variation along the bar $t=60 \mathrm{sec}$ with unequal $E_{S}$

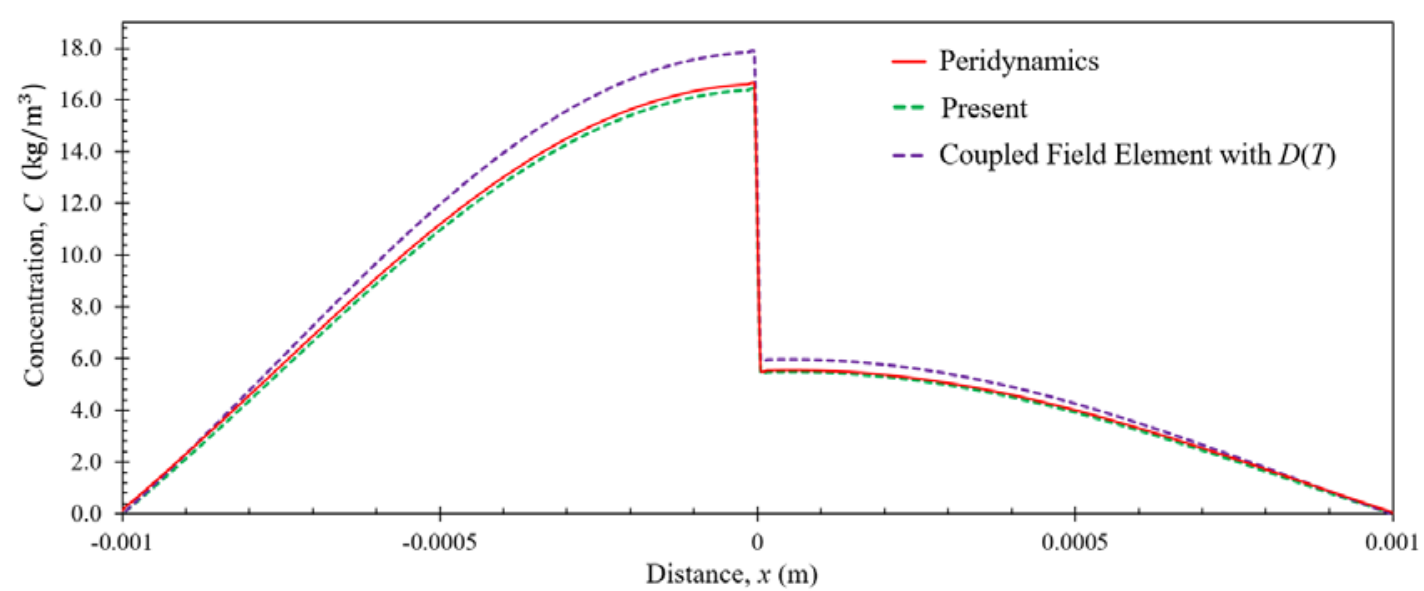

Fig. 7. Moisture concentration variation along the bar $t=60 \mathrm{sec}$ with equal $E_{S}$ 


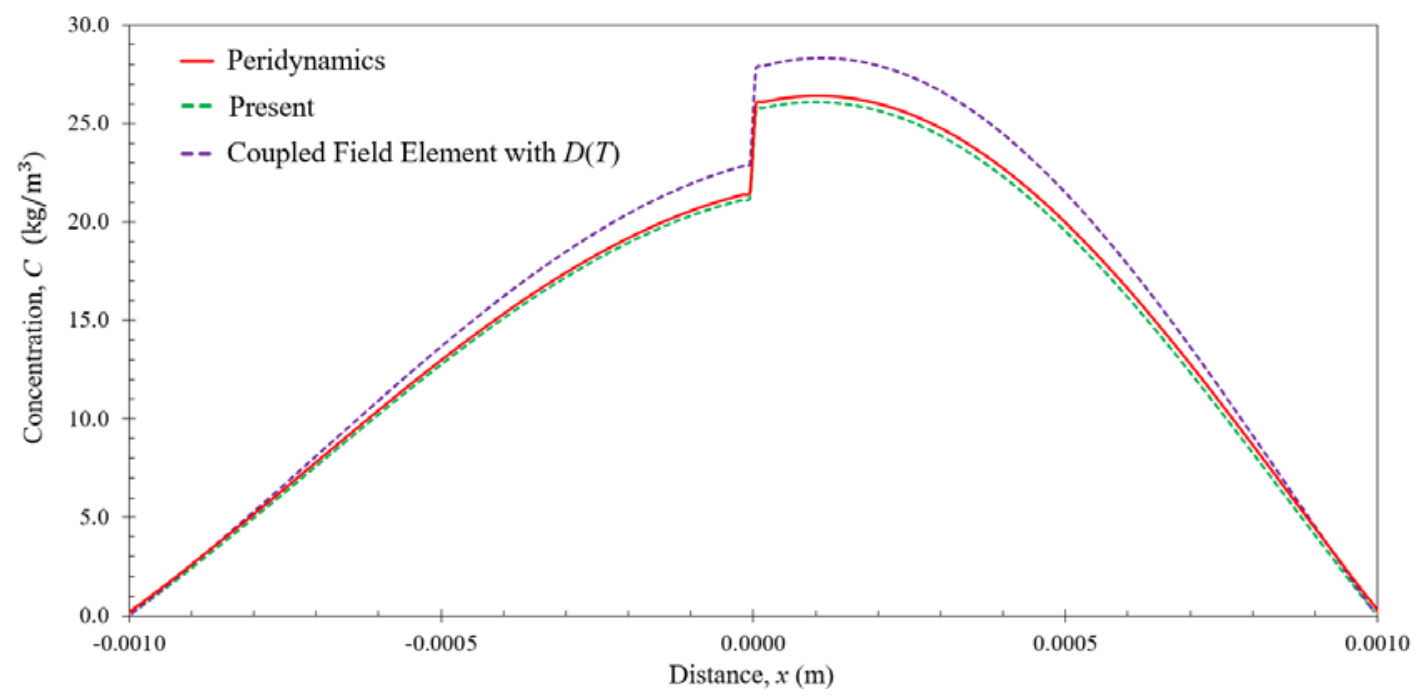

Fig. 8. Moisture concentration variation along the bar $t=60 \mathrm{sec}$ with unequal $E_{S}$ 